\title{
Flavour observables and composite dynamics: leptons
}

\author{
Florian Goertz ${ }^{\mathrm{a}}$ \\ Max-Planck-Institut für Kernphysik, Saupfercheckweg 1, 69117 Heidelberg, Germany
}

Received 16 December 2020 / Accepted 7 July 2021 / Published online 4 August 2021 (C) The Author(s) 2021

\begin{abstract}
We review lepton flavor physics and corresponding observables in the composite Higgs framework with partial compositeness, considering 'UV complete' setups as well as effective and holographic approaches. This includes anarchic flavor setups, scenarios with flavor symmetries, and minimal incarnations of the see-saw mechanism that naturally predict non-negligible lepton compositeness. We focus on lepton flavor violating processes, dipole moments, and on probes of lepton flavor universality, all providing stringent tests of partial compositeness. We discuss the expected size of effects in the different approaches to lepton flavor, which will be useful to understand how a composite lepton sector could look like, given up-to-date experimental constraints.
\end{abstract}

\section{Introduction}

Within the composite Higgs $(\mathrm{CH})$ framework, partial compositeness (PC) offers an attractive means to address the hierarchies present in the flavor sector, while simultaneously suppressing dangerous flavorchaning neutral currents (FCNCs) via a 'geometric' GIM-like mechanism [1-5]. While the basic properties of quarks and leptons share some similarities, such as the large hierarchies in the masses of the charged states, reading

$m_{e}=0.51 \mathrm{MeV} \ll m_{\mu}=106 \mathrm{MeV} \ll m_{\tau}=1.78 \mathrm{GeV}$

in the lepton sector, they also feature striking differences: the leptonic mixing angles are rather sizable [6], all leptons are significantly lighter than the weak scale, and neutrinos are even many orders of magnitude lighter than any other Standard Model (SM) particle, $m_{\nu_{i}} \lesssim 1 \mathrm{eV}$.

Following the paradigm of PC, the lepton masses quoted in Eq. (1) could lead to the expectation that leptons are largely elementary and that the known leptons should behave basically SM-like and for example have a negligible impact on the one-loop Higgs potential or on not extremely well-constrained lepton observables. However, as we will see below, explicit models addressing the structure of charged and neutral lepton masses and mixings can lead to different conclusions and predict a moderate lepton compositeness. Beyond that, even in the case of mostly elementary leptons, the stringent limits from lepton flavor physics can lead to non-trivial bounds on the parameter space.

\footnotetext{
a e-mail: florian.goertz@mpi-hd.mpg.de (corresponding author)
}

PC for leptons has been considered in [7-33], mostly from a low-energy point of view and via holographic methods. More recently, UV complete realizations of partially composite leptons have been envisaged, considering the fundamental degrees of freedom and the dynamics that leads to the bound states that mix linearly with the elementary fermions, which adds structure and potentially correlations to the setups [34-40]. Some promising scenarios, which are dubbed "fundamental partial compositeness" (FPC) [38-40] and will be very briefly reviewed below, assume the fermionic bound states to be composed of an elementary fermion $\mathcal{F}$ and a scalar $\mathcal{S}$, each. The inclusion of scalars has the advantage that the scaling dimension of the corresponding composite operator $\mathcal{O}_{F}$ is expected to vary moderately around the canonical value of $\left[\mathcal{O}_{F}\right]_{0}=[\mathcal{F}]+[\mathcal{S}]=$ $5 / 2$, which would allow to reproduce a viable spectrum of fermion masses via modest anomalous dimensions. While the presence of elementary scalars could reintroduce a hierarchy problem, purely fermionic constructions of PC [34-37] face strong challenges since they require extremely large anomalous dimensions of the composite operators such as to bring their scaling dimension towards $\left[\mathcal{O}_{F}\right] \approx 5 / 2$, starting from a canonical dimension of $3 \times 3 / 2=9 / 2 \gg 5 / 2$. Such large anomalous dimensions seem disfavoured [41-43], which leads in general to too suppressed fermion masses. On the other hand, the approach invoking scalars could be seen as a promising intermediate step to a full UV theory and it would, in fact, be very interesting to construct (potentially more involved) viable models without scalar constituents in the UV, realizing the techni- 
baryons with purely fermionic content and building on the pioneering work of [34-37], see also [44]. ${ }^{1}$

\section{UV realization of partial compositeness for leptons}

Assuming that the composite operators consist of a fermion and a scalar state, $\mathcal{O}_{F}=\langle\mathcal{F} S\rangle$, several models have been studied in the literature, considering the confining technicolor (TC)-like group $G_{\mathrm{TC}}$ to be $\mathrm{SU}\left(N_{\mathrm{TC}}\right), \mathrm{SO}\left(N_{\mathrm{TC}}\right)$, or $\operatorname{Sp}\left(N_{\mathrm{TC}}\right)[38,39,47]$. In the following, we will focus on $G_{\mathrm{TC}}=\operatorname{Sp}\left(N_{\mathrm{TC}}\right)$ with four Weyl fermions $\mathcal{F}^{a}, a=1, . ., 4$, per techni-color, resulting in the global symmetry breaking $(\mathrm{SB})$ pattern $\mathrm{SU}(4)_{\mathcal{F}}$ $\rightarrow \mathrm{Sp}(4)_{\mathcal{F}}$ after formation of the fermionic condensate $[39,47]$

$$
\left\langle\mathcal{F}^{a} \epsilon_{\mathrm{TC}} \mathcal{F}^{b}\right\rangle=\Lambda_{c} f^{2} \Sigma_{\theta}^{a b}
$$

Here $a, b \in \mathrm{SU}(4)_{\mathcal{F}},{ }^{2}$ which contains the SM electroweak (EW) group, $\Lambda_{c} \sim 4 \pi f$ denotes the TC condensation scale (with $f$ the pNGB decay constant), $\epsilon_{\mathrm{TC}}$ is the antisymmetric tensor of $G_{\mathrm{TC}}$, and $\Sigma_{\theta}$ an (antisymmetric) matrix parametrizing the alignment of the vacuum with the $\mathrm{Sp}(4)_{\mathcal{F}}$ group. The techni-fermions are accordingly assumed to form a weak doublet $\mathcal{F}_{1,2}$ with vanishing hypercharge as well as two $S U(2)_{L}$ singlets $\mathcal{F}_{3,4}$ with hypercharges $Y=\mp 1 / 2$, respectively [39,47].

The vacuum matrix can be written as

$$
\Sigma_{\theta}=\cos \theta\left(\begin{array}{ll}
i \sigma_{2} & 0 \\
0 & -i \sigma_{2}
\end{array}\right)+\sin \theta\left(\begin{array}{ll}
0 & \mathbf{1}_{\mathbf{2}} \\
-\mathbf{1}_{\mathbf{2}} & 0
\end{array}\right),
$$

with the $\mathrm{EW}$ vacuum expectation value (vev) $v=$ $\sin \theta f$, such that $\sin \theta=0 \quad(\sin \theta=1)$ corresponds to unbroken (fully broken) EW symmetry. Finally, the pNGBs of the $\mathrm{SU}(4)_{\mathcal{F}} / \mathrm{Sp}(4)_{\mathcal{F}}$ coset are parameterized as fluctuations around this vacuum via the Goldstone matrix

$$
\Sigma(x)=\exp \left[i \frac{2 \sqrt{2}}{f} \Pi_{\hat{a}}(x) T_{\theta}^{\hat{a}}\right] \Sigma_{\theta},
$$

where $T_{\theta}^{\hat{a}}$ are the broken generators of $\mathrm{SU}(4)_{\mathcal{F}} / \mathrm{Sp}(4)_{\mathcal{F}}$, $\Pi_{1,2,3}$ are the EW Goldstone modes, $\Pi_{4}$ is the Higgs boson, and $\Pi_{5}$ an additional EW singlet.

Beyond this, 12 complex scalar degrees of freedom are introduced to realize $\mathrm{FPC}$ with $\left[\mathcal{O}_{F}\right] \approx 5 / 2$, residing in 3 generations of color triplets $\mathcal{S}_{q}$ with hypercharge $Y=-1 / 6$, and corresponding color singlets $\mathcal{S}_{l}$ with

\footnotetext{
1 We refer the reader to $[45,46]$ for a recent interesting construction with heavy scalars.

2 In the absence of fundamental fermion masses, $m_{\mathcal{F}}=0$, the techni-fermions exhibit a global $\mathrm{SU}(4)_{\mathcal{F}}$ symmetry.
}

$Y=1 / 2$. Defining $\mathcal{S}=\left(\mathcal{S}_{q}, \mathcal{S}_{l}\right)^{T}$, the full kinetic and mass terms of the TC sector are thus given by

$$
\begin{aligned}
\mathcal{L}_{\text {kin }}= & -\frac{1}{4} \mathcal{G}_{\mu \nu}^{a} \mathcal{G}^{a \mu \nu}+i \mathcal{F}^{\dagger} \bar{\sigma}^{\mu} D_{\mu} \mathcal{F}-\left(\mathcal{F}^{T} \frac{m_{\mathcal{F}}}{2} \epsilon_{\mathrm{TC}} \mathcal{F}+\text { h.c. }\right) \\
& +\left(D_{\mu} \mathcal{S}\right)^{\dagger}\left(D^{\mu} \mathcal{S}\right)-\mathcal{S}^{\dagger} m_{\mathcal{S}}^{2} \mathcal{S}
\end{aligned}
$$

describing the TC gauge, fermion, and scalar sectors. Note that, arranging the techni-scalars as $\Phi=$ $\left(\mathcal{S},-\epsilon_{T C} \mathcal{S}^{*}\right)^{T}$, in the absence of the mass matrix $m_{\mathcal{S}}$ a global $\mathrm{Sp}(24)_{\mathcal{S}}$ symmetry becomes manifest in the scalar sector, under which $\Phi$ transforms as the fundamental representation. The setup described here is called "minimal fundamental partial compositeness" (MFPC), since it corresponds to the minimal viable amount of techni-matter that leads to a pNGB Higgs and fermionic resonances mixing linearly with the SM fields $[38,39,47]$.

Uplifting the SM-like fermions to spurions, i.e. assigning them transformation properties under the full global symmetry, $\psi^{i}{ }_{a} \equiv(\Psi y)_{a}^{i} \in 24_{\mathcal{S}} \otimes \overline{4}_{\mathcal{F}}$, with $i(a)$ an $\mathrm{Sp}(24)_{\mathcal{S}}\left(\mathrm{SU}(4)_{\mathcal{F}}\right)$ index and $y$ being Yukawa matrices, the Yukawa Lagrangian connecting the TC sector with the SM can be written in compact form as $[39,47]$

$$
-\mathcal{L}_{\text {yuk }}=\psi^{i}{ }_{a} \epsilon_{i j} \Phi^{j} \epsilon_{\mathrm{TC}} \mathcal{F}^{a}+\text { h.c. },
$$

with $\epsilon_{i j}$ the antisymmetric $\operatorname{Sp}(24)_{\mathcal{S}}$ tensor and the elementary fields being embedded in $\psi$. In particular, Eq. (6) contains the UV realization of PC for leptons, reading

$$
\begin{aligned}
& \mathcal{L}_{\text {yuk }} \supset L_{\alpha} y_{L} \mathcal{S}_{l} \epsilon_{\mathrm{TC}} F^{\alpha}-\bar{\nu} y_{\bar{\nu}} \mathcal{S}_{l}^{*} \overline{\mathcal{F}}^{4}+\bar{e} y_{\bar{e}} \mathcal{S}_{l}^{*} \overline{\mathcal{F}}^{3} \\
& -\bar{\nu} \tilde{y}_{\bar{\nu}} \mathcal{S}_{l} \overline{\mathcal{F}}^{3}+\text { h.c. },
\end{aligned}
$$

with the weak doublet $F^{\alpha}=\left(\mathcal{F}^{1}, \mathcal{F}^{2}\right)^{T}$. ${ }^{3}$ Such an explicit UV construction of a $\mathrm{CH}$ with $\mathrm{PC}$ fermions will leave its imprint in flavor and precision observables at low energies, as we will discuss below.

On the other hand, to capture a broader range of potential UV completions for the phenomenological study and in particular to make contact with a large set of previous works on lepton flavor - which were performed in an effective/holographic approach, many of them employing the $\mathrm{SO}(5) / \mathrm{SO}(4)$ coset - we will consider an effective description of $\mathrm{PC}$ in the following. We will thus, in most cases, not specify the microscopic interactions that lead to the linear interactions between elementary fields and composite states. Still, we will comment on specific predictions of the MFPC framework. We also note that even though no fundamental (4D) UV completion for the $\mathrm{CH}$ with elementary fermions exists for the $\mathrm{SO}(5) / \mathrm{SO}(4)$ coset [48], it can be considered as a minimal benchmark model to study the generic features of the $\mathrm{CH}$ setup, where many characteristics are expected to still hold for modified cosets.

\footnotetext{
3 For quarks, a similar Lagrangian emerges, consisting of the first three terms with the replacements $L \rightarrow Q, l \rightarrow$ $q, \nu \rightarrow u, e \rightarrow d$.
} 
Moreover, the mentioned holographic 5D duals [5] can be seen as a completion of the setup, even though, being higher dimensional, ultimately they require their own completion, like string theory [48-53], and the low cutoff on the IR brane leads to only moderately suppressed uncalculable effects.

\section{Effective description: different embeddings of the lepton sector}

At an effective level, below the scale $\Lambda_{\mathrm{UV}}$ where the elementary/composite-sector interactions are generated (Eq. (7) for MFPC), PC for leptons can be described via linear mixings of the SM-like elementary fields with composite operators $\mathcal{O}_{L, R}^{l}$ of the confining sector

$\mathcal{L}_{\text {mix }}=\frac{\lambda_{L}^{\ell}}{\Lambda_{\mathrm{UV}}^{\gamma_{L}^{\ell}}} \bar{l}_{L}^{\ell} \mathcal{O}_{L}^{\ell}+\frac{\lambda_{R}^{\ell}}{\Lambda_{\mathrm{UV}}^{\gamma_{R}^{\ell}}} \bar{l}_{R}^{\ell} \mathcal{O}_{R}^{\ell}+\frac{\lambda_{R}^{\nu_{\ell}}}{\Lambda_{\mathrm{UV}}^{\gamma_{\ell} \gamma_{\ell}}} \bar{\nu}_{R}^{\ell} \mathcal{O}_{R}^{\nu_{\ell}}+$ h.c.,

responsible for their masses. Here, $\ell=e, \mu, \tau$ (with an obvious analogue for quarks), $\lambda_{L, R}^{l}$ are $\mathcal{O}(1)$ couplings and $\gamma_{L, R}^{l}=\left[\mathcal{O}_{L, R}^{l}\right]-5 / 2$ are the anomalous dimensions of the composite-sector operators. Moreover, $l_{L}^{\ell}$, $l_{R}^{\ell}$, and $\nu_{R}^{\ell}$ correspond to the embeddings of the SM-like elementary fields into irreducible representations of the global symmetry of the composite sector, such as $\mathrm{SO}(5)$ in the Minimal Composite Higgs Model (MCHM) [5], according to the representation of the operators they mix with. Treating them as spurions of the full global symmetry, their 'background values' correspond to the SM-like multiplets and the spurious symmetry can be used to estimate their contribution to the Higgs potential via explicit global SB (see, e.g., [53] and below).

For the further analysis, we define the 'degree of compositeness'

$$
\epsilon_{L, R}^{l} \sim \frac{\lambda_{L, R}^{l}}{g_{*}}\left(\mu / \Lambda_{\mathrm{UV}}\right)^{\gamma_{L, R}^{l}}
$$

of a chiral SM-like field, with $g_{*}$ the coupling of the resonances in the strong sector and $\mu \sim \Lambda_{c} \sim \mathcal{O}(\mathrm{TeV})$ the IR scale where the composite sector condenses. Note that in explicit models a SM-like fermion might mix with more than a single type of composite operator. This is for example required for the fundamental representation of $\mathrm{SO}(5)[17,54]$, see also [53], leading to more terms in Eq. (8) (for the fundamental of $\mathrm{SO}(5)$, i.e. the $\mathrm{MCHM}_{5}$, one needs for example $\left.\mathcal{O}_{L}^{\ell}, \mathcal{O}_{L}^{\ell^{\prime}}, \mathcal{O}_{R}^{\ell}, \mathcal{O}_{R}^{\nu \ell}\right){ }^{4}$ Modest differences in the anomalous dimensions $\gamma_{L, R}^{l}$ will automatically lead to a hierarchical spectrum of

\footnotetext{
${ }^{4}$ In the linear mixings we have diagonalized the flavor structure via unitary rotations, which is possible without loss of generality if each elementary fermion mixes only with one composite operator - in the more general case a diagonal form corresponds to an assumption.
}

fermion masses from an anarchic flavor structure in the $\mathrm{UV}$, after integrating out the heavy resonances excited by the operators $\mathcal{O}_{L, R}^{l}$, leading to $m_{\ell} \sim g_{*} v / \sqrt{2} \epsilon_{L}^{\ell} \epsilon_{R}^{\ell} .{ }^{5}$ Also the hierarchical pattern of the CKM matrix in the quark sector follows directly due to a Froggatt-Nielsenlike structure in the mass matrices $[9,55,56]$. Finally, the leptonic mixing matrix with its $\mathcal{O}(1)$ entries, can be obtained, too - with certain assumptions on the model structure, also depending on how neutrino masses are realized [7-18,21-33, 57-59], as we will discuss now.

\subsection{Basic anarchic setup}

Similar to the quark sector, the lepton sector in $\mathrm{CH}$ models can be realized by assuming anarchic values for the dimensionless input parameters, generating the hierarchies in charged-lepton masses after condensation in the composite sector by the UV-scale suppression, $\Lambda_{U V} \gg \mu$, in Eq. (8). The leptonic mixing matrix as well as the neutrino masses, on the other hand, could be kept non-hierarchical by appropriate assumptions on the PC structure. Such scenarios have been envisaged in Refs $[11,14,15,19,23,33]$. In general however, even though there is some suppression of FCNCs from PC, it remains a challenge to evade the very stringent flavor constraints, as we will see below, pushing $f$ above the $\mathrm{TeV}$ scale.

\subsection{Models with flavor symmetries}

There exist several frameworks that refine the fully anarchic approach by invoking flavor symmetries to generate the sought particular form of the leptonic mixing matrix and of neutrino masses together with hierarchical charged-lepton masses and a protection from flavor constraints, going beyond the geometric GIM. Popular flavor groups $G_{f}$ that have been envisaged in the composite Higgs framework are summarized in Table 1. While early constructions focused on $A_{4}, S_{4}$, or the double tetrahedral $T^{\prime}$, the discovery of a non-zero $\theta_{13}$ mixing angle [60-63] lead to a broadening to other (product) groups or to considering spontaneous breaking of such symmetries, which can incorporate this finding. It would be worthwhile to explore such symmetries in a fundamental theory of partial compositeness.

Interestingly, such models with flavor symmetries often feature a suppression of the Yukawa couplings in the composite sector (inducing lepton masses after linear mixing), since those control the magnitude of the breaking of $G_{f}$ (see, e.g., [17]). Moreover, there is a suppression of the left-handed (LH) lepton compositeness, which is bound to be small due to the absence of custodial protection of the $Z \bar{\ell}_{L} \ell_{L}$ couplings. In turn, the $\tau_{R}$ needs to mix quite significantly with the composite sector to generate the moderately large $m_{\tau}$. This can lead to new LHC signatures [20] and interesting effects in Higgs physics $[29,64,65]$, while EW precision

\footnotetext{
${ }^{5}$ It would be interesting to examine the emergence of a
} hierarchical spectrum explicitly in a setup of MFPC. 
Table 1 Popular choices for flavor symmetry $G_{f}$ in the lepton sector, where below $X \in\left\{A_{5}, \Delta(96), \Delta(384)\right\}$

\begin{tabular}{llllllll}
\hline$G_{f}$ & $A_{4} \times Z_{N}$ & $S_{4} \times Z_{N}^{n}$ & $X \times Z_{N}$ & $\Delta(27) \times Z_{4} \times Z_{4}^{\prime}$ & $S_{3}$ & $T^{\prime}$ & $U(N)$ \\
\hline References & {$[13,17,18,25]$} & {$[21,22,26]$} & {$[22]$} & {$[30]$} & {$[28]$} & {$[16]$} & {$[24,33]$} \\
\hline
\end{tabular}

tests (EWPT) can be met due to the custodial protection of right-handed $(\mathrm{RH}) \tau$ couplings. On the other hand, non-negligible compositeness in the charged lepton sector can also emerge beyond such models of flavor symmetries, simply from the scale of neutrino massesnamely in minimal realizations of a seesaw in the $\mathrm{CH}$ framework as we will discuss now.

\subsection{Minimal seesaw model and composite leptons}

In the composite Goldstone-Higgs framework, a very minimal realization of the lepton sector is possible that allows to explain the tiny neutrino masses, by envisaging a type-III seesaw mechanism with heavy fermionic $\mathrm{SU}(2)_{L}$ triplets, which provides at the same time an efficient flavor protection. In fact, if in the MCHM setup neutrino masses are realized via such triplets, a unification of the RH lepton sector is possible and a single, symmetric, representation of $\mathrm{SO}(5)$ can host both the charged $\mathrm{RH}$ leptonic $\mathrm{SU}(2)_{L}$ singlet as well as the $\mathrm{RH}$ seesaw triplet $[29,31,32]$. This leads to a more minimal model for leptons than conventional analogues to the quark sector [17] and the Type-III seesaw construction could also be interesting in the context of leptogenesis, see, e.g., $[66,67]$.

In consequence, the PC Lagrangian in Eq. (8) can be written with only two terms (instead of the usual four) and becomes simply

$$
\mathcal{L}_{\text {mix }}=\frac{\lambda_{L}^{\ell}}{\Lambda_{\mathrm{UV}}^{\gamma_{L}^{\ell}}} \bar{L}_{L}^{\ell} \mathcal{O}_{L}^{\ell}+\frac{\lambda_{R}^{\ell}}{\Lambda_{\mathrm{UV}}^{\gamma_{\mathrm{UV}}^{\ell}}} \bar{L}_{R}^{\ell} \mathcal{O}_{R}^{\ell}+\text { h.c. }
$$

where $L_{L}^{\ell} \sim \mathbf{5}$ and $L_{R}^{\ell} \sim \mathbf{1 4}$, with less degrees of freedom than in minimal leptonic versions of the MCHM envisaged before [17]. In the holographic 5D-dual picture, $L_{L}^{\ell}$ and $L_{R}^{\ell}$ would correspond to bulk fermions reading $[29,31,32]$

$$
\begin{aligned}
& \zeta_{1}^{\ell}=\ell_{1}^{\prime}[-,+] \oplus\left(\begin{array}{cc}
\nu_{1}^{\ell}[+,+] & \tilde{\ell}_{1}[-,+] \\
\ell_{1}[+,+] & \tilde{Y}_{1}^{\ell}[-,+]
\end{array}\right) \text { and } \\
& \zeta_{2}^{\ell}=\ell_{2}^{\prime}[-,-] \oplus\left(\begin{array}{cc}
\nu_{2}^{\ell}[+,-] & \tilde{\ell}_{2}[+,-] \\
\ell_{2}[+,-] & \tilde{Y}_{2}^{\ell}[+,-]
\end{array}\right) \\
& \oplus\left(\begin{array}{ccc}
\hat{\lambda}_{2}^{\ell}[-,-] & \nu_{2}^{\ell \prime \prime}[+,-] & \ell_{2}^{\prime \prime \prime}[+,-] \\
\hat{\nu}_{2}^{\ell}[-,-] & \ell_{2}^{\prime \prime}[+,-] & Y_{2}^{\ell \prime \prime \prime}[+,-] \\
\hat{\ell}_{2}[-,-] & Y_{2}^{\ell \prime \prime}[+,-] & \Theta_{2}^{\ell \prime \prime \prime}[+,-]
\end{array}\right),
\end{aligned}
$$

where we have explicitly shown the decomposition under $\mathrm{SO}(4) \cong \mathrm{SU}(2)_{L} \times \mathrm{SU}(2)_{R}$ : the 5 of $\mathrm{SO}(5)$ decomposes as a singlet and a bi-doublet, the latter represented by a $2 \times 2$ matrix on which the $\mathrm{SU}(2)_{L}\left(\mathrm{SU}(2)_{R}\right)$ transformation acts vertically (horizontally). The $\mathbf{1 4}$ accordingly decomposes as a singlet, bi-doublet, and bi-triplet and both representations feature a charge of $Q_{X}=-1$ under the abelian $\mathrm{U}(1)_{X}$ factor [31]. ${ }^{6}$ The boundary conditions at the two ends of the compactified $A d S_{5}$ space - the UV brane, localized at $z=R \sim M_{\mathrm{Pl}}^{-1}$, and the IR brane, localized at $z=R^{\prime} \sim \mathrm{TeV}^{-1}$, with $z$ the coordinate of the additional spatial dimension $R \leq z \leq R^{\prime}$-are specified in square brackets. A Dirichlet boundary condition for the $\mathrm{RH}(\mathrm{LH})$ chirality is denoted by $[+]([-])$.

Now, the SM-like fields correspond to chiral zero modes of these 5D Dirac fermions, where $\mathrm{LH}(\mathrm{RH})$ zero modes are present for components with $[+,+]([-,-])$ boundary conditions. We can thus identify the $\mathrm{SU}(2)_{L}$ doublet leptons with the zero mode of the left $T_{R}^{3}=$ $+1 / 2$ column in the first bi-doublet, $l_{L}^{\ell} \subset\left(\nu_{1 L}^{\ell}, \ell_{1 L}\right)^{T}$, and the RH charged SM-like leptons as $\ell_{R} \subset \ell_{2}^{\prime}$. In addition, the see-saw triplet resides in the $T_{R}^{3}=+1$ component of the bi-triplet, $\Sigma_{\ell R} \subset\left(\hat{\lambda}_{2 R}^{\ell}, \hat{\nu}_{2 R}^{\ell}, \hat{\ell}_{2 R}\right)^{T}$, see $[31,32]$ for more details. These zero modes will be localized along the extra dimension according to the value of the 5D Dirac-mass $M_{i}$ of the multiplets, parametrized in terms of the dimensionless $c_{i} \equiv M_{i} R$, which correspond to the anomalous dimensions in Eq. (10) as [68] $\gamma_{L, R}^{\ell}=\left|c_{1,2} \mp 1 / 2\right|-1$. UV localization, i.e. $c_{i}<-1 / 2$ $\left(c_{i}>1 / 2\right)$ for LH $(\mathrm{RH})$ zero modes $[1,57,68]$, is identified with elementary fermions in the dual picture, with a suppressed mixing with the composite sector, whereas the more the modes are localized towards the IR brane, the bigger their compositeness.

A (Majorana) mass term is added in the elementary sector, reading

$$
\mathcal{L}_{\mathrm{el}}=-\frac{1}{2}\left[M_{\Sigma}^{\ell \ell^{\prime}} \operatorname{Tr}\left(\bar{\Sigma}_{\ell R}^{c} \Sigma_{\ell^{\prime} R}\right)+\text { h.c. }\right]
$$

with

$$
\Sigma_{\ell}=\left(\begin{array}{cc}
\hat{\nu}^{\ell} / \sqrt{2} & \hat{\lambda}^{\ell} \\
\hat{\ell} & -\hat{\nu}^{\ell} / \sqrt{2}
\end{array}\right), \quad \ell=e, \mu, \tau .
$$

This explains the tiny neutrino masses with $\mathcal{O}(1)$ Yukawa couplings via a large $M_{\Sigma}^{\ell \ell \prime} \gg v$, realizing the (type-III) seesaw mechanism. In the 5D theory, the elementary Majorana mass term corresponds to a brane localized mass for the 5D version of the multiplet $\Sigma_{\ell}\left(\subset \xi_{2}^{\ell}\right)$, simply adding the Lagrangian of Eq. (12) on the UV brane at $z=R$ (where $\mathrm{SO}(4)$ is broken). If now $\Sigma_{\ell}$ would be fully elementary, i.e. the zero modes of $\xi_{2}^{\ell}$ would be localized on the UV brane, it would

\footnotetext{
${ }^{6}$ An additional $\mathrm{U}(1)_{X}$ symmetry is needed to allow for
} viable hypercharges of the SM fermions, with $Y=T_{R}^{3}+Q_{X}$. 
receive an effective Majorana mass of $\mathcal{O}\left(M_{\mathrm{Pl}}\right)$, which is the fundamental scale of the theory (and the warp factor on the UV brane being $a \equiv R / z=1)$. However, this would lead to a significantly too strong suppression of neutrino masses. This calls for a non-negligible compositeness of the $\Sigma_{\ell}$ multiplets, corresponding to a reduced overlap of the $\xi_{2}^{\ell}$ zero modes with the UV brane, such as to warp down the effective 4D Majorana mass significantly below $M_{\mathrm{Pl}}$.

The resulting increased IR localization (i.e., smaller anomalous dimension) of the light modes in $\xi_{2}^{\ell}$, including RH charged leptons with a sizable degree of compositeness, will lead to interesting phenomenological consequences, as discussed further below. Custodial symmetry will still guarantee the agreement of the $Z \bar{\ell}_{R} \ell_{R}$ couplings with measurements [20,64]. On top of that, the picture lined out above allows for a very strong flavor protection, as we will see now.

To avoid dangerous FCNCs, a $\mathrm{U}(3)_{1} \times \mathrm{U}(3)_{2}$ flavor symmetry in the composite sector is envisaged [31,32], corresponding to rotations in flavor space of the $\mathbf{5}$ and the 14 of $\mathrm{SO}(5)$. This is broken by the vev of a spurion $\mathcal{Y}$ in the strong sector, while the purely elementary sector, represented by the Majorana mass term, does not respect the symmetry to start with. The breaking via $\mathcal{Y}$ is necessary in order to induce a massive chiral low-energy spectrum after integrating out the heavy resonances. In the $5 \mathrm{D}$ picture, this corresponds to a breaking of a gauge flavor symmetry $\mathrm{SU}(3)_{1} \times \mathrm{SU}(3)_{2}$ via an IR-brane coupling between the Dirac spinors $\sim \xi_{1}^{\ell} \mathcal{Y} \xi_{2}^{\ell}[31]$, linking the LH and RH SM-like fermions, where

$$
\xi_{1}^{\ell} \sim(\mathbf{3}, \mathbf{1}), \quad \xi_{2}^{\ell} \sim(\mathbf{1}, \mathbf{3}), \quad \mathcal{Y} \sim(\mathbf{3}, \overline{\mathbf{3}}) .
$$

In terms of this source of flavor breaking, the bulk masses (or PC coefficients) can now be expanded as

$$
c_{1}=\eta_{1} \mathbf{1}+\rho_{1} \mathcal{Y Y}^{\dagger}, \quad c_{2}=\eta_{2} \mathbf{1}+\rho_{2} \mathcal{Y}^{\dagger} \mathcal{Y},
$$

with $\eta_{1,2}, \rho_{1,2} \in \mathbb{R}$. This is an appealing picture, since the elementary sector can generate a non-trivial flavor structure, reproducing the leptonic mixing matrix, while the fact that a single spurion breaks the flavor symmetry allows to fully diagonalize the strong sector via a simple unitary rotation. In consequence, no leptonic FCNCs are generated - up to very small effects suppressed by the large Majorana masses.

Regarding the resulting hierarchy of lepton compositeness, it is interesting that actually the electron will feature the largest compositeness, which can be understood from inspecting the scaling of the lepton masses. In fact, the physical charged lepton masses now, in the presence of significant $\mathrm{RH}$ compositeness, scale like $\mathcal{M}_{e} \sim \delta_{\ell \ell^{\prime}} v g_{*} \epsilon_{L}^{\ell}$ (see Eqs. (9) and (10)), which can be understood from the fact that for $\gamma_{R}^{\ell}<0$ a large correction to the $L_{R}^{\ell}$ kinetic term emerges at $\mu=\mathcal{O}(\mathrm{TeV})$, where the conformal sector becomes strongly coupled, such that after canonically normalizing the kinetics, $\epsilon_{R}^{\ell}$ drops out of $\mathcal{M}_{e}$. From the $5 \mathrm{D}$ perspective, this becomes clear realizing that the corresponding wave functions are in a regime where the overlap with the IR brane is no longer exponentially sensitive to the bulk mass. Along the same lines, the neutrino mass matrix reads $\mathcal{M}_{\nu} \sim$ $v^{2} g_{*}^{2} \epsilon_{L}^{\ell} \epsilon_{R}^{\ell}\left(M_{\Sigma}^{\ell \ell^{\prime}}\right)^{-1} \epsilon_{L}^{\ell^{\prime}} \epsilon_{R}^{\ell^{\prime}}$, where the suppression by the elementary Majorana mass $M_{\Sigma}$, expected to reside at the Planck scale, is lifted by the appreciable RH lepton compositeness $\epsilon_{R}^{\ell}$, see also [21]. Since the Dirac masses are suppressed similarly to charged lepton masses, quantitatively this requires entries in the resulting effective Majorana mass matrix even much smaller than $M_{\mathrm{GUT}}$. It now follows directly that realizing hierarchical charged lepton masses and a non-hierarchical neutrino mass matrix requires $\epsilon_{L}^{e} \ll \epsilon_{L}^{\mu} \ll \epsilon_{L}^{\tau} \ll 1$ and $\epsilon_{L}^{\ell} \epsilon_{R}^{\ell} \sim$ constant, and thus $0 \ll \epsilon_{R}^{\tau} \ll \epsilon_{R}^{\mu} \ll \epsilon_{R}^{e}$. This will lead to interesting signatures, like a non-negligible violation of lepton flavor universality (LFU) within the first generations, as we will discuss below.

The appreciable compositeness of leptons in this minimal see-saw model will also have important consequences on the radiatively generated Higgs potential [29]. In fact, since the quantum numbers of the symmetric representation $\mathbf{1 4}$ of $\mathrm{SO}(5)$ allow for the lepton contribution to the Higgs mass to appear at leading order in the degree of compositeness $\epsilon_{R}^{\ell}$, contrary to the general case, large effects are expected even for moderately composite leptons, being actually comparable to the top-quark contribution to $m_{h}$ in the $\mathrm{MCHM}_{5}$, as can be seen in a spurion analysis and also in explicit calculations [29]. By interfering destructively with the top contribution in a sizable region of parameter space, this allows for a larger top-quark breaking of the global symmetry, while still reproducing the light Higgs mass. In turn, the ultra-light top partners that are present in conventional minimal composite Higgs scenarios to reduce the $\mathrm{SO}(5)$ breaking for a fixed $m_{t}$ can be lifted, which will be shown numerically below, see Fig. 1. In this context, note that the additional contribution to the Higgs potential also allows for a viable EWSB in previously excluded most minimal holographic setups for the quark sector (see [29]), where the contribution of the top alone would not be sufficient. This is the case for a fully composite $t_{R}$ singlet, with the LH doublet being embedded in a $\mathbf{5}$, which we will employ in the following, corresponding to $\xi_{U}^{i} \sim \mathbf{5}_{2 / 3}, \xi_{u}^{i} \sim \mathbf{1}_{2 / 3}, \xi_{D}^{i} \sim$ $\mathbf{5}_{-1 / 3}, \xi_{d}^{i} \sim \mathbf{1}_{-1 / 3}, i=1,2,3$, with the subscript denoting $Q_{X}$.

The numerical confirmation of the discussion above has been presented in $[29,31]$. Performing a scan with $\mathcal{O}(1)$ input parameters, fitting the leptonic and quark spectra, the mass of the lightest top partner versus the Higgs mass was evaluated at the compositeness scale, assumed to be $f=1 \mathrm{TeV}$, after accounting for correct EWSB with $v \approx 246 \mathrm{GeV}$. The results are reported as colored points in Fig. 1, with the yellow band being a conservative estimate of the Higgs mass at this scale [29]. We can see from the figure that, in contrast to the conventional $\mathrm{MCHM}_{5}$ (displayed by gray points for comparison), top-partners can be raised significantly 
Fig. 1 Left: mass of the lightest top partner versus $m_{h}$ as a function of the tuning $\Delta_{\mathrm{BG}}$ in the minimal seesaw model with $f=1 \mathrm{TeV}$ (colored points) and corresponding prediction in the $\mathrm{MCHM}_{5}$ (gray points). Right: coefficient of $4 e$ contact operator $c_{e e}$ versus $f$, where the blue (yellow) line shows a fit to the prediction (the upper $95 \%$ C.L. limit)

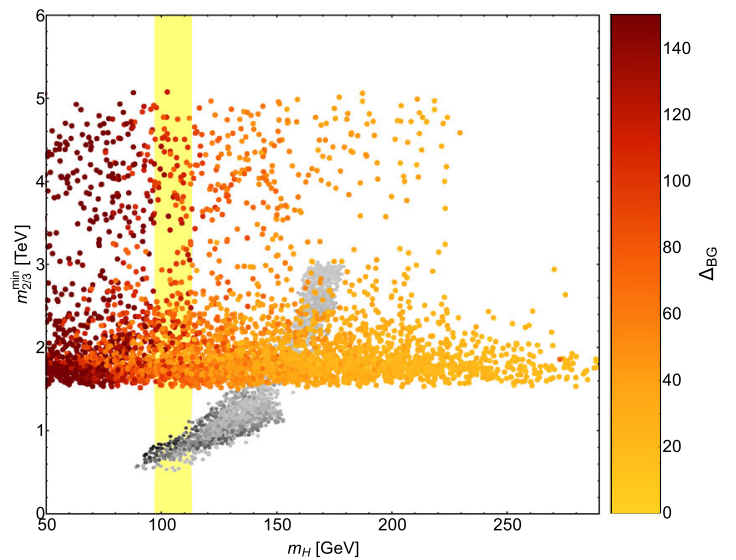

above $f$, reaching up to $5 \mathrm{TeV}$ without blowing up the tuning, measured by the Barbieri-Giudice (BG) prescription [69]. The model thus allows to maintain a small $f \approx 0.8 \mathrm{TeV}$ (as suggested by naturalness arguments, taking into account EWPT), without running into conflict with LHC top partner searches - which are pushing $m_{t^{\prime}} \gtrsim 1.3 \mathrm{TeV}$ [70-72] — and brings us basically back to the tuning already required pre-LHC, see [73]. ${ }^{7}$ On the other hand, the top partner bound corresponds already to $f$ surpassing a TeV in the $\mathrm{MCHM}_{5}[29,74-$ 76], driving the tuning in this model.

While a dedicated survey of bounds on lepton compositeness from flavor and precision physics will be carried out in the next section, a straightforward test of the setup at hand is given by searches for four-lepton contact interactions

$$
\mathcal{O}_{X Y}^{\ell^{1} \ell^{2} \ell^{3} \ell^{4}}=\left(\bar{\ell}_{X}^{1} \gamma_{\mu} \ell_{X}^{2}\right)\left(\bar{\ell}_{Y}^{3} \gamma^{\mu} \ell_{Y}^{4}\right)
$$

with coefficients scaling as $C_{X Y}^{\ell^{1} \ell^{2} \ell^{3} \ell^{4}} \sim \frac{g_{*}^{2}}{m_{*}^{2}} \epsilon_{X}^{\ell^{1}} \epsilon_{X}^{\ell^{2}} \epsilon_{Y}^{\ell^{3}} \epsilon_{Y}^{\ell^{4}}$, where $m_{*}$ is the mass scale of the composite resonances. With the hierarchies quoted before, the largest coefficient is $c_{e e} \equiv C_{R R}^{e e e e}$, which is constrained as $\left|c_{e e}\right|<$ $4 G_{F} / \sqrt{2} \times 0.003$ at $95 \%$ C.L. [77]. The prediction of the minimal seesaw model is shown in the right panel of Fig. 1 in dependence on $f$, where the blue curve corresponds to the best fit to the parameter points and the experimental bound is given by a yellow line. We find that $f=m_{*} / g_{*} \gtrsim 0.9 \mathrm{TeV}$ allows in principle for an agreement with the data in the composite electron scenario.

\section{Predictions and constraints}

In this final section, we will discuss important lepton flavor observables and corresponding predictions in the

\footnotetext{
7 For other setups addressing the issues of light top partners in minimal $\mathrm{CH}$ models, with enlarged representations or by softening the SB, see [74-76].
}

scenarios introduced above. The focus will be on lepton flavor violating (LFV) processes, leptonic dipole moments, as well as LFU violating interactions.

\subsection{Lepton flavor violation and dipole moments}

A stringent test for every model of lepton compositeness is provided by the strong experimental bounds on the decay $\mu \rightarrow e \gamma$, which does not conserve lepton flavor. In composite models, this decay is induced from one-loop penguin diagrams involving heavy resonances $[11,19]$, generating the dipole operators

$$
\mathcal{O}_{\ell \ell^{\prime}}^{\gamma} \equiv e v F_{\mu \nu} \bar{\ell}_{L} \sigma^{\mu \nu} \ell_{R}^{\prime}
$$

with here $\ell=\mu, \ell^{\prime}=e$. The latest experimental limit on the branching ratio $(\mathrm{BR})$ reads $\mathrm{BR}(\mu \rightarrow e \gamma)<4.2 \times$ $10^{-13}$ at $90 \% \mathrm{CL}$ [78], which is significantly stronger than those on the equivalent processes involving the tau lepton, which are $\operatorname{BR}(\tau \rightarrow e \gamma)<3.3 \times 10^{-8}$ and $\operatorname{BR}(\tau \rightarrow \mu \gamma)<4.4 \times 10^{-8}[6]$.

In the anarchic scenario of Sect. 3.1, the BR scales as (see also $[19,23,79,80])$

$$
\begin{gathered}
\operatorname{BR}(\mu \rightarrow e \gamma)=96 \pi^{2} e^{2} \frac{v^{6}}{m_{\mu}^{2}}\left(\left|C_{\mu e}^{\gamma}\right|^{2}+\left|C_{e \mu}^{\gamma}\right|^{2}\right) \\
C_{\mu e}^{\gamma} \sim \frac{1}{16 \pi^{2}} \frac{g_{*}^{3}}{\sqrt{2} m_{*}^{2}} \epsilon_{L}^{\mu} \epsilon_{R}^{e}, \quad C_{e \mu}^{\gamma} \sim \frac{\sqrt{1}}{16 \pi^{2}} \frac{g_{*}^{3}}{\sqrt{2} m_{*}^{2}} \epsilon_{L}^{e} \epsilon_{R}^{\mu} .
\end{gathered}
$$

The experimental constraint quoted before then leads to the bound

$$
\frac{g_{*}^{3}}{m_{*}^{2}} \sqrt{\left|\epsilon_{L}^{\mu} \epsilon_{R}^{e}\right|^{2}+\left|\epsilon_{L}^{e} \epsilon_{R}^{\mu}\right|^{2}} \lesssim \frac{10^{-7}}{\mathrm{TeV}^{2}}
$$

Employing $m_{\ell} \sim g_{*} v / \sqrt{2} \epsilon_{L}^{\ell} \epsilon_{R}^{\ell}$, we can derive a stringent conservative limit on the resonance scale by setting $\epsilon_{R}^{\ell}=\epsilon_{L}^{\ell}$, which becomes

$$
\frac{m_{*}}{g_{*}} \gtrsim 20 \mathrm{TeV} \quad(\mathrm{BR}(\mu \rightarrow e \gamma))
$$




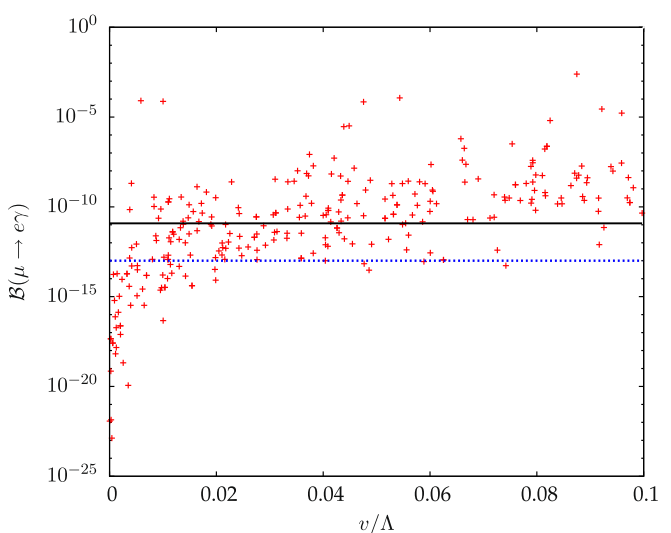

Fig. 2 Left: prediction for $\operatorname{BR}(\mu \rightarrow e \gamma)$ in the $\mathrm{MCHM}_{5^{-}}$ like setup with $A_{4}$ flavor symmetry versus the $A_{4}$ breaking vev (over the cutoff) $v / \Lambda$ in the elemenatry sector, scanning the parameter space with $m_{*} \sim 3 \mathrm{TeV}, g_{*} \sim 4$ (from [17]).

agreeing with the results given in $[33,79]$ to good accuracy. It is evident that the fully anarchic scenario is pushed to rather large mass scales due to this boundeven for modest $g_{*} \sim 4$ the resonances would be expected to reside well beyond the reach of current or near-future colliders. The constraints from flavorchanging tau decays on the resonance scale can be obtained straightforwardly from the above expressions, but are a factor of $\gtrsim 10$ weaker.

If new $\mathrm{CP}$ violation is present due to the strong sector, another powerful bound arises from the electric dipole moment $(\mathrm{EDM}) d_{e}$ of the electron, which is proportional to the imaginary part of the diagonal $(1,1)$ component of the dipole coefficient, see Eq. (17), and scales as [33,79]

$$
d_{e} \sim \operatorname{Im}\left(c_{e}\right) \frac{e}{16 \pi^{2}} \frac{g_{*}^{3}}{m_{*}^{2}} \epsilon_{L}^{e} \epsilon_{R}^{e} \frac{v}{\sqrt{2}},
$$

with $c_{e}$ containing the (assumed $\mathcal{O}(1)$ ) phase of the anarchic setup. The recent stringent limit of the ACME collaboration of $d_{e}<1.1 \times 10^{-29} e \mathrm{~cm}$ [81] thus constrains (again for $\epsilon_{R}^{\ell}=\epsilon_{L}^{\ell}$ )

$$
\frac{m_{*}}{g_{*}} \gtrsim 75 \mathrm{TeV} \quad\left(\mathrm{eEDM}, \operatorname{Im}\left(c_{e}\right) \sim 1\right)
$$

which agrees well with the limits presented in [33,79], after updating the bounds used there to the latest ACMEII limit. The corresponding dipole moments for the muon or the tau do not lead to meaningful current constraints. Moreover, the limit above depends crucially on the amount of $\mathrm{CP}$ violation in the new sector.

Finally, bounds from $\mu-e$ conversion in Gold constrain the flavor-changing couplings to the $\mathrm{Z}$ boson, which are sensitive to the combinations $\epsilon_{L}^{\mu} \epsilon_{L}^{e}$ and $\epsilon_{R}^{\mu} \epsilon_{R}^{e}$. Setting once more $\epsilon_{R}^{\ell}=\epsilon_{L}^{\ell}$, the SINDRUMII 90\% CL limit of $\Gamma(\mu A u \rightarrow e A u) / \Gamma_{\text {capture }}(\mu A u)<7 \times 10^{-13}$

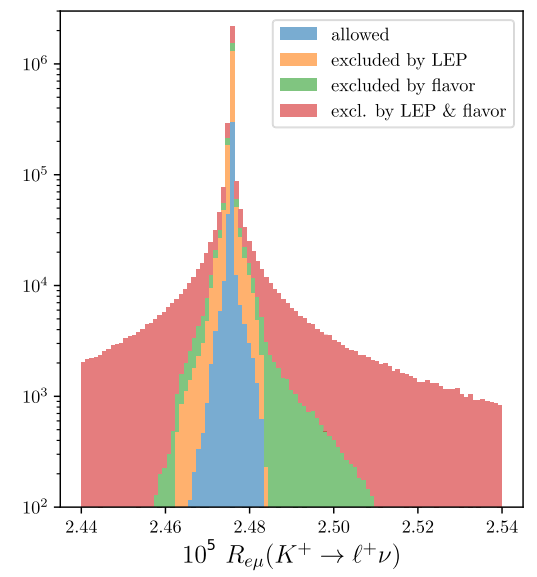

The solid (dashed) line shows the old MEGA (projected MEG) limit. Right: histogram of predictions for the ratio $R_{e \mu}$ after a parameter space scan in the MFPC scenario (adapted from [47]), see text for details

[82] puts a bound on the resonance scale of (see $[33,79])$

$$
\frac{m_{*}}{\sqrt{g_{*}}} \gtrsim 3 \mathrm{TeV} \quad(\mu A u \rightarrow e A u)
$$

This is significantly weaker than the exclusions above and the bound from $\mu \rightarrow$ eee transitions, probing the same operator, is another factor $\sim 3$ less constraining. Similar decays involving the $\tau$ lepton are even less constraining, and the same holds true for the anomalous magnetic moment of the muon, see [33] for a recent overview.

In composite scenarios with a protection due to flavor symmetries, discussed in Sect. 3.2, the bounds from LFV quoted above are typically very much weaker, and vary over the different models considered in Tab. 1. For example, in models with an $A_{4}$ symmetry the limits can be reduced to the $1 \mathrm{TeV}$ scale. In such setups, the nontrivial transformation properties of $\mathrm{LH}$ doublets and $\mathrm{RH}$ neutrinos under $A_{4}$ in flavor space $[13,17]$ lead to flavor-universal PC mixing parameters for the corresponding multiplets. On the other hand, the sector of charged RH leptons is diagonal, with the different flavors living in different singlet representations of $A_{4}$, but non-universal. In turn, after spontaneous breaking of $A_{4}$ in the elementary and confining sectors, to leading order this allows to rotate to a basis where the full flavor structure is diagonal, up to Majorana masses in the elementary sector. This induces a powerful suppression of LFV such that resonances around $m_{*} \approx 3 \mathrm{TeV}$ are in agreement with limits from LFV for a typical strongly coupled sector with $g_{*} \approx 4[17]$ (see also [25]). This is shown more quantitatively in the left panel of Fig. 2, where for small $A_{4}$ breaking, parametrized by the breaking vev over the cutoff $v / \Lambda$, most of the points are in agreement with a bound of $\operatorname{BR}(\mu \rightarrow e \gamma)<5 \times 10^{-13}$. Similar statements hold true for the minimal seesaw model of Sect. 3.3, where 
LFV does not impose stringent constraints after invoking a similar flavor protection, as discussed in detail in Sect. 3.3.

Turning to minimal 4D UV completions of PC, considering the MFPC scenario as introduced in Sect. 2, it is interesting to see whether additional predictions emerge just from the UV setup, leading to (8) at low energies. First of all, in the MFPC construction no custodial protection for $Z \bar{f} f$ couplings is present [38], which (depending on further structure) limits the size of potentially observable effects in flavor physics due to stringent constraints from $Z$-pole measurements at LEP, pushing the compositeness scale. Still, interesting effects are possible, as we will see below. Moreover, with additional ingredients such a protection could be achieved [38] and a further investigation of this issue, in particular in the context of lepton compositeness and flavor observables discussed here, would be interesting.

Besides, now the flavor structure in the effective theory is induced by the corresponding properties of the fundamental constituents. If for example the scalar mass matrix in Eq. (5) has a flavor-trivial form, $m_{\mathcal{S}}^{2} \sim$ $\mathbf{1}$, as can in fact emerge if the TC scalars acquire their mass solely from strong TC interactions (and the TCscalar potenital conserves flavor) [38], important consequences arise. The coefficient of the dipole operator in Eq. (17) will be proportional to the corresponding SMfermion Yukawa matrix, $C_{\ell \ell^{\prime}}^{\gamma} \propto y_{\ell \ell^{\prime}}^{\mathrm{SM}}$, to leading approximation, and thus diagonal and real in the same basis. This allows to push both the $\operatorname{BR}(\mu \rightarrow e \gamma)$ and the electron dipole moment $d_{e}$ below the experimental limit, even for TC scales below a $\mathrm{TeV}$ and $g_{*} \sim 4 \pi$ [38], i.e.,

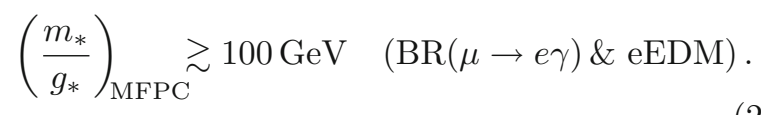

\subsection{Lepton flavor universality violation}

We now turn to processes that are diagonal in lepton flavor, but might be flavor non-universal. In particular, we will focus mostly on tests of LFU between the first and second generation in neutral-current $B$ decays, parametrized by the ratios

$$
\begin{aligned}
R_{K} & \equiv \frac{\mathrm{BR}\left(B^{+} \rightarrow K^{+} \mu^{+} \mu^{-}\right)}{\mathrm{BR}\left(B^{+} \rightarrow K^{+} e^{+} e^{-}\right)}, \\
R_{K^{*}} & \equiv \frac{\mathrm{BR}\left(B^{0} \rightarrow K^{*} \mu^{+} \mu^{-}\right)}{\operatorname{BR}\left(B^{0} \rightarrow K^{*} e^{+} e^{-}\right)}
\end{aligned}
$$

and explored extensively from the experimental side at LHCb [84-86] and Belle $[87,88]$. Due to the PC mechanism of mass generation, a certain level of LFU violation is in fact a generic prediction of models with $\mathrm{PC}$ leptons, even in the absence of LFV, with potentially measurable effects being well motivated in some setups. The current experimental results and (dominant) statistical uncertainties for the dilepton invariant mass squared within $1.1<q^{2} / \mathrm{GeV}^{2}<6$ read $R_{K}=$
$0.846_{-0.054}^{+0.060}$ and $R_{K^{*}}=0.69_{-0.07}^{+0.11}[85,86]$, and strongly disfavor $R_{K, K^{*}}>1$, where in the SM $R_{K}=R_{K^{*}}=1$, up to $1 \%$ effects due to $m_{e} \neq m_{\mu}{ }^{8}$

In the $\mathrm{CH}$ framework, crucial operators in this context are four-fermion contact terms, induced from exchange of heavy EW resonances, reading (c.f. Eq. (16))

$$
\mathcal{O}_{X Y}^{q^{1} q^{2} \ell^{1} \ell^{1}}=\left(\bar{q}_{X}^{1} \gamma_{\mu} q_{X}^{2}\right)\left(\bar{\ell}_{Y}^{1} \gamma^{\mu} \ell_{Y}^{2}\right)
$$

with coefficients scaling as $C_{X Y}^{q^{1} q^{2} \ell^{1} \ell^{2}} \sim \frac{g_{*}^{2}}{m_{*}^{2}} \epsilon_{X}^{q^{1}} \epsilon_{X}^{q^{2}} \epsilon_{Y}^{\ell^{1}} \epsilon_{Y}^{\ell^{2}}$ and becoming important for sizable compositeness [31, 33,47,83,89-91]. Whether these can lead to a good agreement with the measurements above sensitively depends on the compositeness of the various fermion chiralities. In fact, taken together, the LHCb results already significantly constrain a potential sizable compositeness of $\mathrm{RH}$ muons in general (if some $b-s$ current talks non-negligibly to the strong sector, i.e. $\epsilon_{X}^{s} \epsilon_{X}^{b}>0$ ) and of LH muons or electrons in combination with a somewhat composite RH $b-s$ current [83]. From general arguments, moving into the quadrant of $R_{K, K^{*}}<1$ requires either LH muon or electron compositeness and basically LH $b-s$ compositeness or RH electron compositeness, irrespectively of the $b-s$ chirality [83] (see also $[89,92])$. For example, focusing on LH muons or $\mathrm{RH}$ electrons, we find that the single-coefficient best fit solutions [92-94] could be achieved with

$$
\frac{g_{*}^{2}}{m_{*}^{2}} \epsilon_{L}^{s} \epsilon_{L}^{b} \epsilon_{L}^{\mu} \epsilon_{L}^{\mu} \sim \frac{10^{-3}}{\mathrm{TeV}^{2}} \quad \text { or } \quad \frac{g_{*}^{2}}{m_{*}^{2}} \epsilon_{X}^{s} \epsilon_{X}^{b} \epsilon_{R}^{e} \epsilon_{R}^{e} \sim 4 \cdot \frac{10^{-3}}{\mathrm{TeV}^{2}},
$$

where $X=L, R$. Trying to realize one of these patterns led to some efforts in the $\mathrm{CH}$ community, envisaging for example enhanced muon compositeness [33, 47, 89-91].

In $M F P C$, this would correspond to sizable fundamental Yukawa couplings in the LH muon sector, i.e. large $\left(y_{L}\right)_{22}$ in Eq. (7) (sticking to a lepton-flavor diagonal structure to leading order, similarly to as discussed in Sect. 4.1), while in turn $\left(y_{\bar{e}}\right)_{22}$ would be tiny in order not to make the muon to heavy. Further important constraints on scenarios with sizable LH compositeness are given by strong bounds on deviations in charged current semi-leptonic kaon and pion decays, which are known to better than percent level, like the ratio $R_{e \mu}\left(K^{+} \rightarrow\right.$ $\left.\ell^{+} \nu\right) \equiv \mathrm{BR}\left(K^{+} \rightarrow e^{+} \nu\right) / \mathrm{BR}\left(K^{+} \rightarrow \mu^{+} \nu\right)$. Moreover, stringent constraints on the partial width of the $Z$ boson are particularly relevant for the MFPC scenario, lacking custodial protection. ${ }^{9}$ The right panel of Fig. 2 shows a histogram of a scan in the MFPC setup [47], after imposing bounds from meson-antimeson mixing. The yellow and green points are excluded from $Z$-boson partial width and flavor constraints (semileptonic charged current decays), respectively, while the

\footnotetext{
${ }^{8}$ The recent results from Belle $[87,88]$ feature still a sizable uncertainty and are compatible both with the SM and the LHCb findings employed here.

9 As this prevents solving the $R_{D^{(*)}}$ anomalies [39], it would be interesting to address.
} 


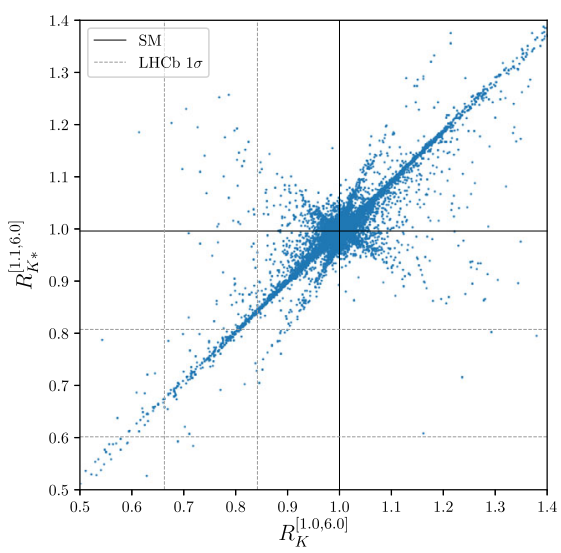

Fig. 3 Left: prediction in the $R_{K}-R_{K^{*}}$ plane in the MFPC scenario, varying $1 \mathrm{TeV}<f<3 \mathrm{TeV}$ (from [47]), see text for details. Right: corresponding viable points in the

red points violate both, see [47] for more details. Keeping only viable points, finally the left panel of Fig. 3 displays the prediction in the $R_{K}-R_{K^{*}}$ plane after a full scan of the MFPC model [47]. We can see that the best fit values can be reached by a set of viable points, and similar conclusions were drawn in the holographic $[90,91]$ and $4 \mathrm{D}$ effective [89] incarnations of PC.

Finally, while it is appealing that $\mathrm{CH}$ models are flexible enough to account for the found tendency, it would also be interesting to find a motivated setup that makes a clearer prediction on the form of LFU violation, increasing the opportunities to probe it. In fact, the minimal seesaw model of Sect. 3.3 furnishes such a setup since due to its minimal and unifying character, as explained there, it unambiguously predicts a $\mathrm{RH}$ electron compositeness as the dominating effect, leading straightforwardly to $R_{K, K^{*}}<1$. Ending up in any other quadrant in this plane would exclude the model, as can be seen from the scan provided in the right panel of Fig. 3. On the other hand a good fit of $R_{K, K^{*}} \sim 0.8$ is easily obtained while keeping the deviation in the $B_{s}$ mass difference $\Delta M_{B_{s}}$ - which is generated due to $\epsilon_{X}^{s} \epsilon_{X}^{b}>0$ and indicated by the shades of blue - at an acceptable $\lesssim 20 \%$ level. In addition, the parameter space is not significantly restricted from measurements of charged current decays like $R_{e \mu}\left(K^{+} \rightarrow \ell^{+} \nu\right)$ or $\operatorname{BR}(\pi \rightarrow e \nu)$, since the LH leptons in the minimal seesaw model are very elementary. In light of the custodial protection of $\mathrm{RH} Z$ couplings and the discussed flavor protecion, the most stringent bound is in fact expected from the $4 e$ contact interactions, which is fulfilled for the given scan with $f=1.2 \mathrm{TeV}$ (see Sect. 3.3). Moreover, it is interesting to note that the updated constraint on $\Delta M_{B_{s}}$ from Ref. [95] poses a strong challenge to significantly reducing $R_{K^{(*)}}$ via LH muon compositeness. A similar reduction due to $\mathrm{RH}$ electron compositeness remains viable, since it is consistent with effects both in LH and RH quark currents, as discussed before, which can cancel to some extent in $\Delta M_{B_{s}}$. We conclude noting that, as becomes clear form the discus-

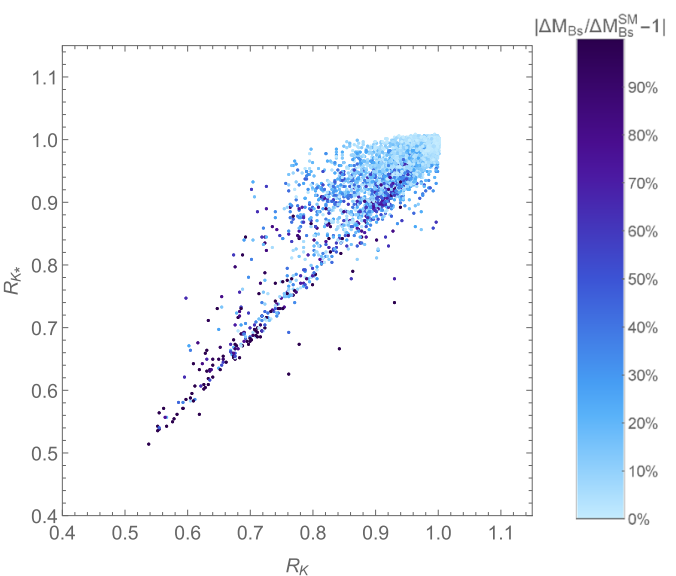

minimal seesaw model [83] with $f=1.2 \mathrm{TeV}$, where the color code displays deviations in the $B_{s}$ mass difference from the SM prediction

sions above, also in the case that with more statistics the fit might move towards $R_{K, K^{*}} \approx 1$, tests of LFU violation remain a crucial part of the program to probe $\mathrm{PC}$ in general.

\section{Conclusions}

We have reviewed lepton flavor physics in composite Higgs models with partial compositeness, both from a fundamental and an effective point of view. After discussing the particularities of different approaches to lepton flavor, including anarchic setups, scenarios with flavor symmetries, and unified see-saw models, motivating non-negligible lepton compositeness, we reviewed their phenomenology and confronted them with various measurements. Our discussion included observables sensitive to lepton flavor violation, to $\mathrm{CP}$ violation, and to lepton flavor universality violation, pointing out crucial differences between the models, including varying limits on the compositeness scale. In fact, different patterns of predictions for these observables, combined with results from searches for (potentially light) resonances, offer a promising means to get a handle on the nature of leptons, after a potential establishment of deviations from SM predictions, or further increased limits.

Acknowledgements I am grateful to Simone Blasi, Giacomo Cacciapaglia, Adrián Carmona Bermúdez, and Csaba Csáki for recent useful conversations with links to the discussed topics.

Funding Open Access funding enabled and organized by Projekt DEAL.

Open Access This article is licensed under a Creative Commons Attribution 4.0 International License, which permits use, sharing, adaptation, distribution and reproduction in any medium or format, as long as you give appropriate credit 
to the original author(s) and the source, provide a link to the Creative Commons licence, and indicate if changes were made. The images or other third party material in this article are included in the article's Creative Commons licence, unless indicated otherwise in a credit line to the material. If material is not included in the article's Creative Commons licence and your intended use is not permitted by statutory regulation or exceeds the permitted use, you will need to obtain permission directly from the copyright holder. To view a copy of this licence, visit http://creativecomm ons.org/licenses/by/4.0/.

\section{References}

1. T. Gherghetta, A. Pomarol, Bulk fields and supersymmetry in a slice of AdS. Nucl. Phys. B 586, 141-162 (2000). arXiv:hep-ph/0003129

2. S.J. Huber, Q. Shafi, Fermion masses, mixings and proton decay in a Randall-Sundrum model. Phys. Lett. B 498, 256-262 (2001). arXiv:hep-ph/0010195

3. K. Agashe, A. Delgado, M.J. May, R. Sundrum, RS1, custodial isospin and precision tests. JHEP 08, 050 (2003). arXiv:hep-ph/0308036

4. K. Agashe, G. Perez, A. Soni, Flavor structure of warped extra dimension models. Phys. Rev. D 71, 016002 (2005). arXiv:hep-ph/0408134

5. K. Agashe, R. Contino, A. Pomarol, The minimal composite Higgs model. Nucl. Phys. B 719, 165-187 (2005). arXiv:hep-ph/0412089

6. P.A. Zyla et al., Review of particle physics. PTEP 2020(8), $083 \mathrm{C} 01$ (2020)

7. S.J. Huber, Q. Shafi, Neutrino oscillations and rare processes in models with a small extra dimension. Phys. Lett. B 512, 365-372 (2001). arXiv:hep-ph/0104293

8. S.J. Huber, Q. Shafi, Majorana neutrinos in a warped 5-D standard model. Phys. Lett. B 544, 295-306 (2002). arXiv:hep-ph/0205327

9. S.J. Huber, Flavor violation and warped geometry. Nucl. Phys. B 666, 269-288 (2003). arXiv:hep-ph/0303183

10. G. Moreau, J.I. Silva-Marcos, Neutrinos in warped extra dimensions. JHEP 01, 048 (2006). arXiv:hep-ph/0507145

11. K. Agashe, A.E. Blechman, F. Petriello, Probing the Randall-Sundrum geometric origin of flavor with lepton flavor violation. Phys. Rev. D 74, 053011 (2006). arXiv:hep-ph/0606021

12. G. Perez, L. Randall, Natural neutrino masses and mixings from warped geometry. JHEP 01, 077 (2009). arXiv:0805.4652

13. C. Csaki, C. Delaunay, C. Grojean, Y. Grossman, A model of lepton masses from a warped extra dimension. JHEP 10, 055 (2008). arXiv:0806.0356

14. K. Agashe, T. Okui, R. Sundrum, A common origin for neutrino anarchy and charged hierarchies. Phys. Rev. Lett. 102, 101801 (2009). arXiv:0810.1277

15. K. Agashe, Relaxing constraints from lepton flavor violation in 5D flavorful theories. Phys. Rev. D 80, 115020 (2009). arXiv:0902.2400
16. M. Chen, K.T. Mahanthappa, Yu. Felix, A viable Randall-Sundrum model for quarks and leptons with T-prime family symmetry. Phys. Rev. D 81, 036004 (2010). arXiv:0907.3963

17. F. del Aguila, A. Carmona, J. Santiago, Neutrino masses from an A4 symmetry in holographic composite Higgs models. JHEP 08, 127 (2010). arXiv:1001.5151

18. A. Kadosh, E. Pallante, An A(4) flavor model for quarks and leptons in warped geometry. JHEP 08, 115 (2010). arXiv:1004.0321

19. C. Csaki, Y. Grossman, P. Tanedo, Y. Tsai, Warped penguin diagrams. Phys. Rev. D 83, 073002 (2011). arXiv:1004.2037

20. F. del Aguila, A. Carmona, J. Santiago, Tau Custodian searches at the LHC. Phys. Lett. B 695, 449-453 (2011). arXiv: 1007.4206

21. C. Hagedorn, M. Serone, Leptons in holographic composite Higgs models with non-abelian discrete symmetries. JHEP 10, 083 (2011). arXiv:1106.4021

22. C. Hagedorn, M. Serone, General lepton mixing in holographic composite Higgs models. JHEP 02, 077 (2012). arXiv: 1110.4612

23. B. Keren-Zur, P. Lodone, M. Nardecchia, D. Pappadopulo, R. Rattazzi, L. Vecchi, On partial compositeness and the $\mathrm{CP}$ asymmetry in charm decays. Nucl. Phys. B 867, 394-428 (2013). arXiv:1205.5803

24. G. von Gersdorff, M. Quiros, M. Wiechers, Neutrino mixing from Wilson lines in warped space. JHEP 02 079 (2013). arXiv:1208.4300

25. A. Kadosh, $\Theta_{1} 3$ and charged lepton flavor violation in "warped" $A_{4}$ models. JHEP 06, 114 (2013). arXiv: 1303.2645

26. G.-J. Ding, Y.-L. Zhou, Dirac neutrinos with $S_{4}$ flavor symmetry in warped extra dimensions. Nucl. Phys. B 876, 418-452 (2013). arXiv:1304.2645

27. M. Redi, Leptons in composite MFV. JHEP 09, 060 (2013). arXiv:1306.1525

28. M. Frank, C. Hamzaoui, N. Pourtolami, M. Toharia, Unified flavor symmetry from warped dimensions. Phys. Lett. B 742, 178-182 (2015). arXiv:1406.2331

29. A. Carmona, F. Goertz, A naturally light Higgs without light top partners. JHEP 05, 002 (2015). arXiv: 1410.8555

30. P. Chen, G.-J. Ding, D. Alma, C.A.V.-A. Rojas, J.W.F. Valle, Warped flavor symmetry predictions for neutrino physics. JHEP 01, 007 (2016). arXiv:1509.06683

31. A. Carmona, F. Goertz, Lepton flavor and nonuniversality from minimal composite Higgs setups. Phys. Rev. Lett. 116(25), 251801 (2016). arXiv:1510.07658

32. A. Carmona, F. Goertz, A flavor-safe composite explanation of $R_{K}$. Nucl. Part. Phys. Proc. 285-286, 93-98 (2017). arXiv:1610.05766

33. M. Frigerio, M. Nardecchia, J. Serra, L. Vecchi, The bearable compositeness of leptons. JHEP 10, 017 (2018). arXiv:1807.04279

34. J. Barnard, T. Gherghetta, T.S. Ray, UV descriptions of composite Higgs models without elementary scalars. JHEP 02, 002 (2014). arXiv:1311.6562

35. G. Ferretti, D. Karateev, Fermionic UV completions of composite Higgs models. JHEP 03, 077 (2014). arXiv:1312.5330 
36. G. Ferretti, UV completions of partial compositeness: the case for a SU(4) Gauge group. JHEP 06, 142 (2014). arXiv:1404.7137

37. L. Vecchi, A dangerous irrelevant UV-completion of the composite Higgs. JHEP 02, 094 (2017). arXiv: 1506.00623

38. F. Sannino, A. Strumia, A. Tesi, E. Vigiani, Fundamental partial compositeness. JHEP 11, 029 (2016). arXiv:1607.01659

39. G. Cacciapaglia, H. Gertov, F. Sannino, A.E. Thomsen, Minimal fundamental partial compositeness. Phys. Rev. D 98(1), 015006 (2018). arXiv:1704.07845

40. A. Agugliaro, F. Sannino, Real and complex fundamental partial compositeness. JHEP 07, 166 (2020). arXiv:1908.09312

41. T. DeGrand, Y. Shamir, One-loop anomalous dimension of top-partner hyperbaryons in a family of composite Higgs models. Phys. Rev. D 92(7), 075039 (2015). arXiv:1508.02581

42. C. Pica, F. Sannino, Anomalous dimensions of conformal Baryons. Phys. Rev. D 94(7), 071702 (2016). arXiv:1604.02572

43. V. Ayyar, T. DeGrand, D.C. Hackett, W.I. Jay, E.T. Neil, Y. Shamir, B. Svetitsky, Partial compositeness and baryon matrix elements on the lattice. Phys. Rev. D 99(9), 094502 (2019). arXiv:1812.02727

44. D.B. Franzosi, G. Ferretti, Anomalous dimensions of potential top-partners. SciPost Phys. 7(3), 027 (2019). arXiv: 1905.08273

45. G. Cacciapaglia, S. Vatani, C. Zhang, Composite Higgs Meets Planck Scale: partial compositeness from partial unification. 11 (2019). arXiv:1911.05454

46. G. Cacciapaglia, S. Vatani, C. Zhang, The Techni-PatiSalam composite Higgs. 5 2020. arXiv:2005.12302

47. F. Sannino, P. Stangl, D.M. Straub, A.E. Thomsen, Flavor physics and flavor anomalies in minimal fundamental partial compositeness. Phys. Rev. D 97(11), 115046 (2018). arXiv:1712.07646

48. G. Cacciapaglia, C. Pica, F. Sannino, Fundamental composite dynamics: a review. Phys. Rep. 877, 1-70 (2020). arXiv:2002.04914

49. N. Arkani-Hamed, S. Dimopoulos, G.R. Dvali, The hierarchy problem and new dimensions at a millimeter. Phys. Lett. B 429, 263-272 (1998). arXiv:hep-ph/9803315

50. I. Antoniadis, N. Arkani-Hamed, S. Dimopoulos, G.R. Dvali, New dimensions at a millimeter to a Fermi and superstrings at a TeV. Phys. Lett. B 436, 257-263 (1998). arXiv:hep-ph/9804398

51. L. Randall, R. Sundrum, A large mass hierarchy from a small extra dimension. Phys. Rev. Lett. 83, 3370-3373 (1999). arXiv:hep-ph/9905221

52. B. Bellazzini, C. Csáki, J. Serra, Composite Higgses. Eur. Phys. J. C 74(5), 2766 (2014). arXiv:1401.2457

53. G. Panico, A. Wulzer, The composite NambuGoldstone Higgs, vol. 913. (Springer, Berlin, 2016). arXiv:1506.01961

54. R. Contino, L. Da Rold, A. Pomarol, Light custodians in natural composite Higgs models. Phys. Rev. D 75, 055014 (2007). arXiv:hep-ph/0612048

55. S. Casagrande, F. Goertz, U. Haisch, M. Neubert, T. Pfoh, Flavor physics in the Randall-Sundrum model: I.
Theoretical setup and electroweak precision tests. JHEP 10, 094 (2008). arXiv:0807.4937

56. C. Csaki, A. Falkowski, A. Weiler, The flavor of the composite pseudo-Goldstone Higgs. JHEP 09, 008 (2008). arXiv:0804.1954

57. Y. Grossman, M. Neubert, Neutrino masses and mixings in nonfactorizable geometry. Phys. Lett. B 474, 361-371 (2000). arXiv:hep-ph/9912408

58. T. Gherghetta, Dirac neutrino masses with Planck scale lepton number violation. Phys. Rev. Lett. 92, 161601 (2004). arXiv:hep-ph/0312392

59. G. Cacciapaglia, M. Rosenlyst. Loop-generated neutrino masses in composite Higgs models. 10 (2020). arXiv:2010.01437

60. K. Abe et al., Indication of electron neutrino appearance from an accelerator-produced off-axis muon neutrino beam. Phys. Rev. Lett. 107, 041801 (2011). arXiv:1106.2822

61. F.P. An et al., Observation of electron-antineutrino disappearance at Daya Bay. Phys. Rev. Lett. 108, 171803 (2012). arXiv:1203.1669

62. J.K. Ahn et al., Observation of reactor electron antineutrino disappearance in the RENO experiment. Phys. Rev. Lett. 108, 191802 (2012). arXiv:1204.0626

63. P. Adamson et al., Measurement of neutrino and antineutrino oscillations using beam and atmospheric data in MINOS. Phys. Rev. Lett. 110(25), 251801 (2013). arXiv:1304.6335

64. A. Carmona, F. Goertz, Custodial leptons and Higgs decays. JHEP 04, 163 (2013). arXiv:1301.5856

65. A. Carmona, F. Goertz, Composite Taus and Higgs decays. PoS EPS-HEP2013, 267 (2013). arXiv: 1310.3825

66. C.H. Albright, S.M. Barr, Leptogenesis in the type III seesaw mechanism. Phys. Rev. D 69, 073010 (2004). arXiv:hep-ph/0312224

67. S. Mishra, Neutrino mixing and leptogenesis with modular $S_{3}$ symmetry in the framework of type III seesaw. 8 (2020). arXiv:2008.02095

68. R. Contino, A. Pomarol, Holography for fermions. JHEP 11, 058 (2004). arXiv:hep-th/0406257

69. R. Barbieri, G.F. Giudice, Upper bounds on supersymmetric particle masses. Nucl. Phys. B 306, 63-76 (1988)

70. M. Aaboud et al., Combination of the searches for pairproduced vector-like partners of the third-generation quarks at $\sqrt{s}=13 \mathrm{TeV}$ with the ATLAS detector. Phys. Rev. Lett. 121(21), 211801 (2018). arXiv:1808.02343

71. A.M. Sirunyan et al., Search for vector-like quarks in events with two oppositely charged leptons and jets in proton-proton collisions at $\sqrt{s}=13 \mathrm{TeV}$. Eur. Phys. J. C 79(4), 364 (2019). arXiv:1812.09768

72. A.M. Sirunyan et al., Search for pair production of vectorlike quarks in the fully hadronic final state. Phys. Rev. D 100(7), 072001 (2019). arXiv:1906.11903

73. F. Goertz, Composite Higgs theory. PoS ALPS2018, 012 (2018). arXiv:1812.07362

74. G. Panico, M. Redi, A. Tesi, A. Wulzer, On the tuning and the mass of the composite Higgs. JHEP 03, 051 (2013). arXiv:1210.7114

75. S. Blasi, F. Goertz, Softened symmetry breaking in composite Higgs models. Phys. Rev. Lett. 123(22), 221801 (2019). arXiv:1903.06146 
76. S. Blasi, C. Csaki, F. Goertz. A natural composite Higgs via universal boundary conditions. 4 (2020). arXiv:2004.06120

77. M. Raidal et al., Flavour physics of leptons and dipole moments. Eur. Phys. J. C 57, 13-182 (2008). arXiv:0801.1826

78. A.M. Baldini et al., Search for the lepton flavour violating decay $\mu^{+} \rightarrow \mathrm{e}^{+} \gamma$ with the full dataset of the MEG experiment. Eur. Phys. J. C 76(8), 434 (2016). arXiv:1605.05081

79. K. Agashe, M. Bauer, F. Goertz, S.J. Lee, L. Vecchi, L.T. Wang, Y. Felix, Constraining RS Models by future flavor and collider measurements: a snowmass whitepaper. 10 (2013). arXiv:1310.1070

80. G.F. Giudice, C. Grojean, A. Pomarol, R. Rattazzi, The strongly-interacting light Higgs. JHEP 06, 045 (2007). arXiv:hep-ph/0703164

81. V. Andreev et al., Improved limit on the electric dipole moment of the electron. Nature 562(7727), 355-360 (2018)

82. W.H. Bertl et al., A Search for muon to electron conversion in muonic gold. Eur. Phys. J. C 47, 337-346 (2006)

83. A. Carmona, F. Goertz, Recent $B$ physics anomalies: a first hint for compositeness? Eur. Phys. J. C 78(11), 979 (2018). arXiv:1712.02536

84. R. Aaij et al., Test of lepton universality using $B^{+} \rightarrow$ $K^{+} \ell^{+} \ell^{-}$decays. Phys. Rev. Lett. 113, 151601 (2014). arXiv:1406.6482

85. R. Aaij et al., Test of lepton universality with $B^{0} \rightarrow K^{* 0} \ell^{+} \ell^{-}$decays. JHEP 08, 055 (2017). arXiv:1705.05802

86. R. Aaij et al., Search for lepton-universality violation in $B^{+} \rightarrow K^{+} \ell^{+} \ell^{-}$decays. Phys. Rev. Lett. 122(19), 191801 (2019). arXiv:1903.09252

87. A. Abdesselam et al, Test of lepton flavor universality in $B \rightarrow K^{*} \ell^{+} \ell^{-}$decays at Belle. 4 (2019). arXiv:1904.02440

88. A. Abdesselam et al., Test of lepton flavor universality in $B \rightarrow K \ell^{+} \ell^{-}$decays. 8 (2019). arXiv:1908.01848

89. C. Niehoff, P. Stangl, D.M. Straub, Violation of lepton flavour universality in composite Higgs models. Phys. Lett. B 747, 182-186 (2015). arXiv:1503.03865
90. E. Megias, G. Panico, O. Pujolas, M. Quiros, A natural origin for the LHCb anomalies. JHEP 09, 118 (2016). arXiv: 1608.02362

91. E. Megias, M. Quiros, L. Salas, Lepton-flavor universality violation in $\mathrm{R}_{K}$ and $R_{D^{(*)}}$ from warped space. JHEP 07, 102 (2017). arXiv:1703.06019

92. G. D'Amico, M. Nardecchia, P. Panci, F. Sannino, A. Strumia, R. Torre, A. Urbano, Flavour anomalies after the $R_{K} *$ measurement. JHEP 09, 010 (2017). arXiv: 1704.05438

93. W. Altmannshofer, P. Stangl, D.M. Straub, Interpreting hints for lepton flavor universality violation. Phys. Rev. D 96(5), 055008 (2017). arXiv:1704.05435

94. J. Aebischer, W. Altmannshofer, D. Guadagnoli, M. Reboud, P. Stangl, D.M. Straub, B-decay discrepancies after Moriond 2019. Eur. Phys. J. C 80(3), 252 (2020). arXiv:1903.10434

95. L. Di Luzio, M. Kirk, A. Lenz, Updated $B_{s}$-mixing constraints on new physics models for $b \rightarrow s \ell^{+} \ell^{-}$anomalies. Phys. Rev. D 97(9), 095035 (2018). arXiv:1712.06572 